\title{
Pushing the limits of reconditioning: extended normothermic lung perfusion in an extended criteria donor
}

\author{
Marco Schiavon $^{1}$, Davide Zampieri ${ }^{1}$, Giuseppe Marulli ${ }^{1}$, Enrico Verderi ${ }^{1}$, Alessandro Rebusso ${ }^{1}$, Giovanni \\ Maria Comacchio ${ }^{1}$, Samuele Nicotra ${ }^{1}$, Monica Loy ${ }^{1}$, Francesca Lunardi ${ }^{2}$, Paolo Feltracco ${ }^{3}$, Fiorella \\ Calabrese $^{2}$, Emanuele Cozzi ${ }^{4}$, Federico Rea ${ }^{1}$ \\ ${ }^{1}$ Thoracic Surgery Division, Department of Cardiac, Thoracic, Vascular Sciences and Public Health, ${ }^{2}$ Pathology Division, Department of Cardiac, \\ Thoracic, Vascular Sciences and Public Health, ${ }^{3}$ Institute of Anesthesiology and Intensive Care, Department of Medicine, ${ }^{4}$ Transplant Immunology \\ Unit, Department of Cardiac, Thoracic and Vascular Sciences and Public Health, Padova University Hospital, Padova, Italy \\ Correspondence to: Marco Schiavon, MD, PhD. Thoracic Surgery Division, Department of Cardiac, Thoracic, Vascular Sciences and Public Health, \\ University of Padova, Via Giustiniani, 2, 35128 Padova, Italy. Email: marco.schiavon@unipd.it.
}

Submitted Jun 01, 2018. Accepted for publication Oct 08, 2018.

doi: $10.21037 /$ jtd.2018.10.125

View this article at: http://dx.doi.org/10.21037/jtd.2018.10.125

\section{Introduction}

Lung transplantation is the best therapeutic option in selected patients with end-stage respiratory diseases; its wider application, however, is precluded by the shortage of available organs. Among the strategies to increase the pool of usable lungs, the introduction of ex vivo lung perfusion (EVLP) systems is one of the most exciting and promising realities $(1,2)$. These systems, emerged as an effective tool for the assessment of donors after cardiac death (DCDs) (3), have evolved to allow the preservation and reconditioning of extended criteria lungs by extending the period of $e x$ vivo perfusion, either in a static or portable fashion (4). The portable devices used in clinical practice [Organ Care System (OCS) (Transmedics, Andover, MA, USA)] usually limit ex vivo perfusion to a 10-12-hour maximum window. These time-intervals and the perfusion strategies may be effective for recruiting maneuvers and for assessment of most standard and extended-criteria lung donors, however the extension of such an observation period could be crucial for three main reasons. First, several organ alterations (such as edema, infection or suspicion of aspiration) may require longer reconditioning/monitoring time; second, several logistic issues (procurement complications, long-distance donor-hospital) may benefit from an extended preservation. Finally, new therapeutic approaches such as mesenchymal stromal cell, gene and pharmacological therapy may require longer ex vivo periods to be effective.

In this article, we report a successfully prolonged lung perfusion period for up to 18 hours due to procurement and implant-related complications in a critical high-LAS patient.

\section{Case presentation}

In January 2016, a 58-year-old female (blood group: 0-, BMI 24.6) was listed for bilateral lung transplantation as a consequence of end-stage hypersensitivity pneumonitis with a Lung Allocation Score (LAS) of 47.6. The patient signed written informed consent for lung transplantation and for data publication. In March 2017, the patient was admitted to our hospital because of development a left pneumothorax with acute respiratory failure, requiring chest tube insertion; the procedure transiently improved respiratory failure. The patient's LAS score increased to 80.8 due to the development of pulmonary hypertension [median pulmonary artery pressure (mPAP), $40 \mathrm{mmHg}$ ] and the need of total assistance for daily activity. A suitable lung donor was assigned to our center only one month later and located almost 1,000 $\mathrm{km}$ away from our hospital. The donor was a 34-year-old male who died from an intracranial bleeding after 5 days of hospitalization. Bronchoscopy showed the presence of moderate secretions, chest $\mathrm{X}$-ray revealed slight infiltrates at the right lower lobe of the donor (Figure 1) and $\mathrm{PaO}_{2} / \mathrm{FiO}_{2}$ ratio was $450 \mathrm{mmHg}$, leading to an Oto score of 5 (5) and a UMN-DLQI score of 40 (6). Among the clinical information collected at time of donation, the most relevant was the 

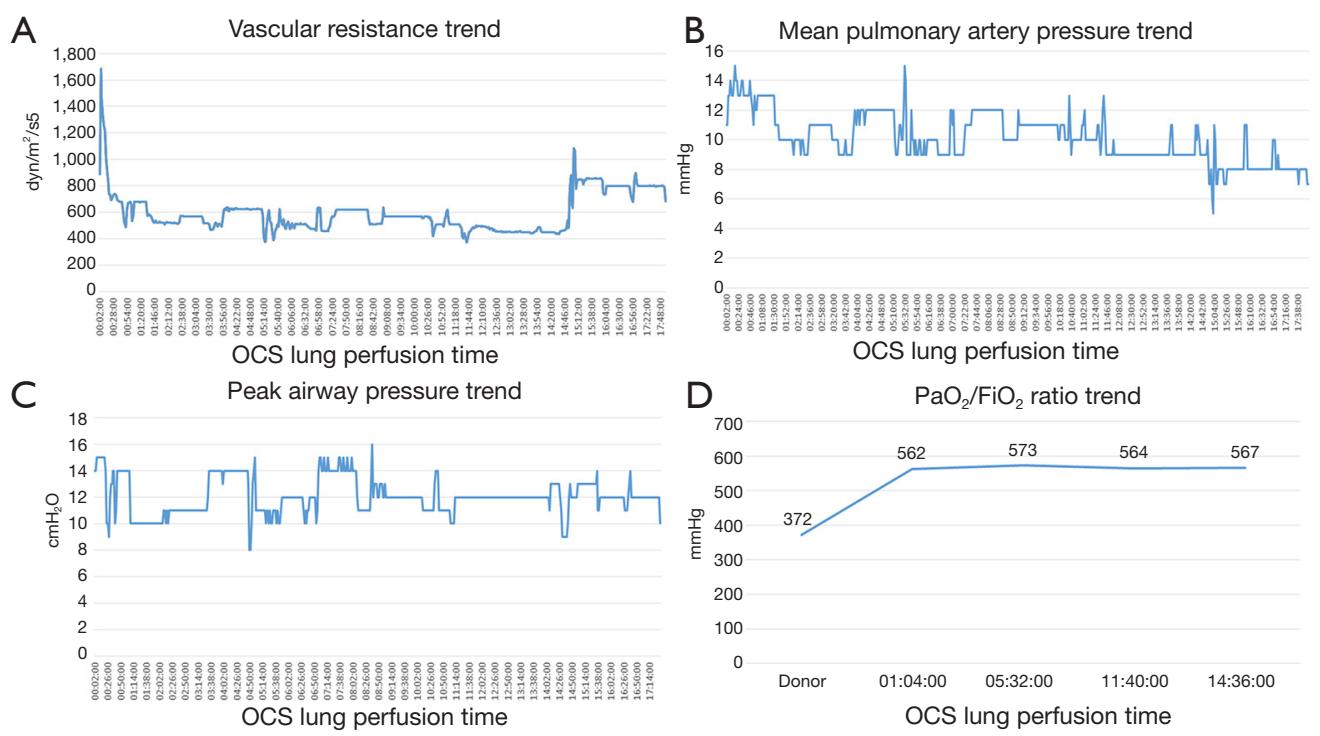

Figure 1 OCS lung parameters during normothermic perfusion showing a trend to reduction of vascular resistance (A) and pulmonary pressure (B) and a stability of peak airway pressure (C). Finally, $\mathrm{PaO}_{2} / \mathrm{FiO}_{2}$ ratio (D) increased from donor to OCS evaluation. Interestingly, lung oxygenation increased during OCS perfusion with a final value of $567 \mathrm{mmHg}$. OCS, Organ Care System.

presence of a stage IV chronic renal failure due to type 1 diabetes mellitus requiring hemodialysis for the last 4 years.

To reduce the risk of lung edema, the donor underwent hemodialysis before procurement. Nevertheless, after sternotomy, lung inspection revealed edematous organs, in particular at the lower lobes, and the presence of two small nodules in the left lower lobe. Arterial blood gas, after recruitment maneuvers of atelectatic areas, revealed a $\mathrm{PaO}_{2} /$ $\mathrm{FiO}_{2}$ ratio of $372 \mathrm{mmHg}$. Due to the inability to perform a timely histological evaluation at the donation site the decision was taken to proceed with the organ procurement using the OCS for donor lung edema reduction, and the risk of organ worsening during cold ischemic transport (7), postponing the histological analysis of the pulmonary nodules upon arrival at the Padua hospital. All procedures were performed according to the ISHLT ethics guidelines, the study was approved by the Institutional Review Board.

The lungs were flushed and cooled with $6 \mathrm{~L}(5 \mathrm{~L}$ anterogradely supplemented with $50 \mathrm{mg}$ nitroglycerine and 1 L retrograde) of Perfadex (XVIVO perfusion, Goteborg, Sweden), procured in standard way and connected to the system 69 min after aortic clamp. Normothermic perfusion with packed red blood cells (pRBCs) was performed according to the company guidelines. After stabilization of both perfusion and ventilation of the graft, a bronchoscopy was performed at the donor's site that excluded the presence of secretions. Moreover, an initial oxygenation capacity of donors' lungs was assessed, revealing a $\mathrm{PaO}_{2} / \mathrm{FiO}_{2}$ ratio of $562 \mathrm{mmHg}$. Once arrived at the recipient hospital, a frozen section incision biopsy of the two lung nodules was performed directly on the device, with closure of the surrounding visceral pleura with two stiches without any air leaks. The final diagnosis reported the presence of necrotizing granulomas, highly suspicious of tuberculosis. At the same time, a second functional analysis $\left(\mathrm{PaO}_{2} /\right.$ $\mathrm{FiO}_{2}$ ratio of $571 \mathrm{mmHg}$ ) and an $\mathrm{X}$-ray of the lung graft inside the OCS, performed as previously described (8), confirmed the satisfactory quality of preservation (Figure 1), with a significant reduction of lung edema and a good quality of lung aeration (Figure 2). After consultation with an expert infectious disease physician and following adequate patient informed consent, the decision was taken to proceed with transplantation. As the recipient had severe pulmonary hypertension and because of long organ reconditioning, a peripheral femoral-femoral arterialvenous $(\mathrm{A}-\mathrm{V}) \mathrm{ECMO}$ was established from the start of the surgical procedure. Following the first pneumonectomy, the two lungs were divided in the organ chamber and the left lung, cold flushed, was successfully implanted after $14 \mathrm{~h} 54 \mathrm{~min}$ of normothermic perfusion (cold ischemic time $189 \mathrm{~min}$ ). The right lung was kept in the machine with adjusted parameters for single lung (setting tidal 
volume at $250 \mathrm{~mL} / \mathrm{min}$, and pump flow at $0.8 \mathrm{~L} / \mathrm{min}$ ) for further $3 \mathrm{~h} 4 \mathrm{~min}$. After $17 \mathrm{~h} 58 \mathrm{~min}$ of total perfusion, the right lung was implanted (cold ischemic time $192 \mathrm{~min}$ ). Altogether, the second lung remained 21 hours outside the donor body. After removal from the OCS system and before implantation, a lung biopsy was performed in the right middle lobe (pre-reperfusion sample). An additional small resection of the left lower lobe, which included the two nodules, was performed at the end of procedure (postreperfusion sample), just before the closure of the chest. The two biopsies were sent to the pathologist to evaluate

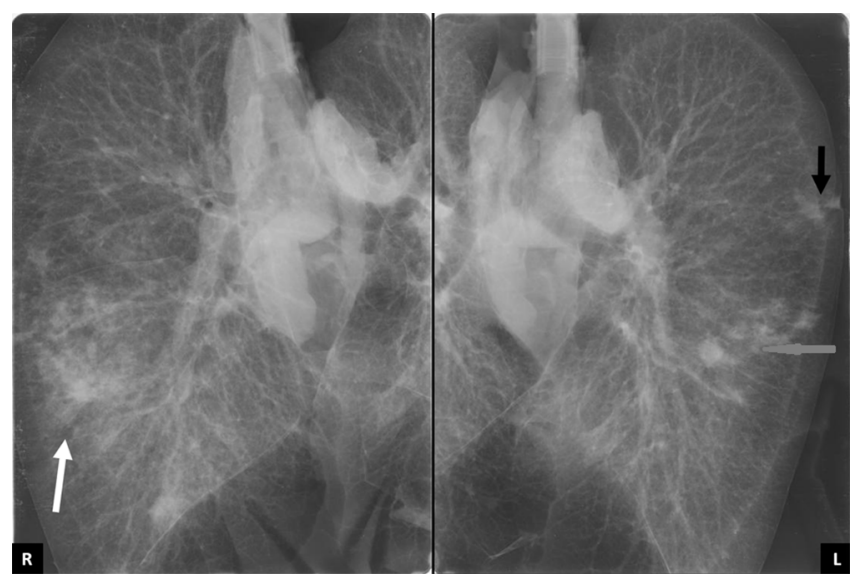

Figure 2 Lung X-ray performed 7 hours after OCS lung perfusion showing residual sign of lung edema in the right lower lobe (white arrow), the presence of two small lung nodules in the left lower lobe near the fissure (black arrow) and some isolated congestive areas in the left para-hilar lung (grey arrow). OCS, Organ Care System. both the infectious suspicion and assess the ischemia/ reperfusion injury (Figure 3). Pre-reperfusion tissue sample did not show any sign of edema, but only the presence of mild congestion and severe granulocyte margination. In the post-reperfusion sample moderate edema, severe congestion and granulocyte margination were observed. The nodule lesions were confirmed as foci of necrotizing granulomas, etiologically related to Mycobacterium tuberculosis (a finding corroborated by PCR analysis) for which a specific treatment was initiated in the early postoperative period. At the end of the transplant procedure A-V ECMO was continued in the ICU due to the persistence of pulmonary hypertension and hemodynamic instability requiring significant cardioactive support. Chest $\mathrm{X}$-ray initially displayed interstitial lung congestion, and Primary Graft Dysfunction (PGD) scores at 0, 24, 48, 72 hours were 3, 3, 2 and 2, respectively. The improvement of both chest $\mathrm{X}$-ray and gas exchange, the reduction of PAP values, a better right ventricle- pulmonary artery coupling, along with improvement of left ventricular function, allowed for ECMO removal on post-operative day 2. An initially moderate reduction in glomerular filtration rate, followed by marked oliguria, required a prolonged continuous venovenous hemofiltration $(\mathrm{CVVH})$ and finally intermittent dialysis treatment. A percutaneous tracheostomy was performed on p.o. day 3 , not because of abnormalities of gas exchange but as a consequence of significant respiratory muscle atrophy and early complete muscle exhaustion at first attempts to wean her off mechanical ventilation. Full mechanical ventilation was required for 45 days, which was followed by nocturnal ventilator assistance for another
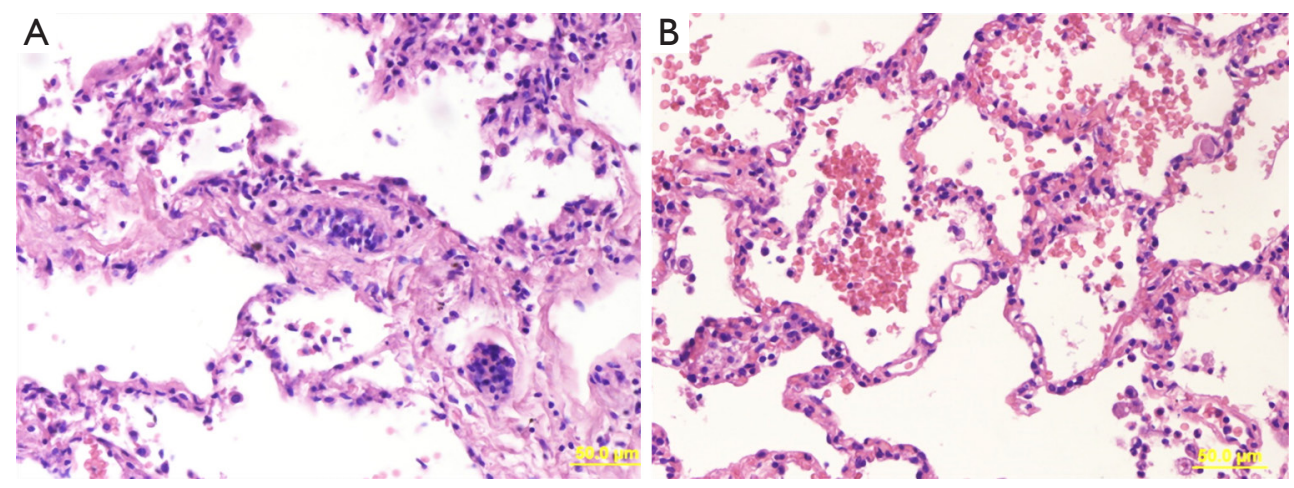

Figure 3 Histologic evaluation of pre- and post-reperfusion lung samples. (A) Haematoxylin and eosin stained section of pre-reperfusion tissue sample showing absence of edema/congestion and presence of moderate granulocyte margination. (B) Haematoxylin and eosin stained section of post-reperfusion tissue sample showing moderate congestion associated with interalveolar blood extravasation and moderate mixed interstitial inflammatory cell infiltration. 
15 days. Tracheal decannulation was performed on p.o. day 70 , after 10 days of complete spontaneous ventilation. She was discharged from the ICU on day 72 , and transferred to the pneumology department to complete her physical rehabilitation. The patient was discharged from hospital on day 132 in satisfactory conditions, with continued dialysis treatment but without ventilator support.

\section{Discussion}

Extending the duration of normothermic perfusion is a desirable new frontier of EVLP (9). Different ex vivo systems, both static and portable, have proven in several experiences $(1,2)$ to preserve the lungs in a physiological status, and limit the damage arising as a consequence of cold ischemia. This ability allows for the evaluation and improvement of the characteristics of the organ, in the socalled "reconditioning process" that in clinical practice usually takes about 4-6 hours.

The introduction of the OCS lung system in the setting of organ procurement has given a first impulse to prolong the perfusion time as this device, created for preservation purposes, combines the evaluation and reconditioning of organs soon at the donor hospital. However, in the various clinical applications in ideal donors, the OCS ex vivo period of perfusion does not differ much from the classical 6 hours $(10,11)$. Although the Expand trial, which implies use of OCS for extended-criteria donors, has enrolled lung donors with an EVLP interval limit of up to 12 hours (12), to our knowledge, the longest ex vivo perfusion period in humans has been reported by Ceulemans et al. (13) with 11 hours of perfusion/ventilation that enabled successful combined lung-liver transplant.

At present, the maximum safe preservation time obtainable with EVLP remains unknown. Indeed, nobody has yet established the golden hours of an EVLP timecourse, in which the device gain is at its best for lung, and beyond which it is reasonable to expect a worsening in graft function.

In 2008, Cypel (14) demonstrated the functional stability of a 12-hour lung perfusion in a pig model using an acellular solution. The Minnesota group (15) conducted a swine prolonged (up to 24 hours) perfusion study on the OCS lung device with 3 different perfusates, namely acellular buffered dextran-albumin, isolated RBCs and whole blood solution. The study showed that the use of autologous whole blood perfusion allowed for 24 hours preservation without functional deterioration and was found to be significantly superior to RBCs for maintaining stability of all monitored parameters, in particular after the first 12 hours. On the contrary, acellular perfusion could not be extended beyond 6 hours due to prohibitively high vascular resistance, edema, and worsening lung compliance. More recently, the Hannover group (16) conducted a similar perfusion study using Steen solution (acellular or enriched with erythrocytes) and low-potassium dextran (LPD) solution (mostly, but not completely, similar to OCS solution, used in clinical setting for OCS lung instrumentation) adding erythrocytes. The most favourable outcome was observed in organs utilizing Steen solution enriched with erythrocytes as perfusate, whereas the least favourable outcome was seen with LPD solution enriched with erythrocytes for perfusion.

Taken together, pre-existing data generated in the pig suggest that EVLP instrumented with dextran and RBCs might not be compatible with successful reconditioning beyond $8-12$ hours.

In this paper, we report for the first time a case of prolonged normothermic perfusion that substantially exceeds 12 hours with subsequent successful graft transplantation. In this case, the impending deterioration of the recipient clinical conditions (development of pulmonary hypertension, prolonged immobilization and respiratory failure), that would have precluded any further prolongation of the waiting-list time, forced us to proceed with transplantation. In addition, the logistic and clinical circumstances led us to extend lung preservation beyond the known critical time-point. Unquestionably, the clinical management of this patient turned out to be quite challenging, with most of the post-transplant complications likely related to her critical pre-operative status. In particular, the prolonged immobilization, due to persistent pneumothorax, resulted in an irreversible muscular fatigue syndrome with very arduous and prolonged post-operative weaning from mechanical ventilation. In addition, peri-operative pulmonary hypertension requiring intense cardioactive support and ECMO, represented an important contributing factor for post-operative kidney injury and PGD. The consequent renal failure requiring dialytic treatment is a well-recognized cause of difficult fluid and immunosuppression management, lung interstitial congestion, cardiovascular complications and superinfections (17).

Whereas the publication of the results of the INSPIRE trial showed that there is a significant reduction in the rate of PGD in standard criteria donors (18) with the use 
of OCS Lung compared to standard cold preservation, it is still under investigation whether this protective effect of OCS Lung may be present also in marginal donors. In our case, the high PGD rates could be mostly related to the prolonged ECMO support and the critical preoperative conditions of the patients (particularly the nearly complete muscle exhaustion at the time of transplantation) rather than the prolonged organ perfusion. For all these reasons, in this case the PGD rate could not represent the most reliable indicator of the capability of the system to guarantee an optimal preservation of the organ for a long time. It is noteworthy, indeed, that throughout the OCS lung procedure all functional, radiological as well as morphological parameters confirmed an adequate organ preservation. The increased congestion score after reperfusion is a well-known sign of ischemia-reperfusion injury (19); however, compared to the other parameters (edema and granulocyte margination) is more affected by alterations related to surgical biopsy and histological procession.

In addition, an additional improvement in vascular resistance, airways and pulmonary pressure beyond 12-hour preservation was observed. Similarly, the lung gas-exchange capacity remained satisfactory over time with a final assessment at 14 hours confirming a $\mathrm{PaO}_{2} / \mathrm{FiO}_{2}$ ratio above $560 \mathrm{mmHg}$.

In conclusion, our experience demonstrates that EVLP procedures can likely be extended beyond 12 hours and up to 18 hours allowing the safe management of time-requiring clinical cases. The real time-limit of normothermic perfusion, however, has yet to be defined and warrants further investigations.

\section{Acknowledgements}

None.

\section{Footnote}

Conflicts of Interest: The authors have no conflicts of interest to declare.

Informed Consent: Witten informed consent was obtained from the patient for the writing of this manuscript.

\section{References}

1. Wang JQ, Gu WP, Deng QQ, et al. Endothelial progenitor cell miR-126 promotes homing of endothelial progenitor cells within arterial thrombus in patients with cerebral infarction and its molecular mechanism. Eur Rev Med Pharmacol Sci 2018;22:1078-83.

2. Cypel M, Yeung JC, Liu M, et al. Normothermic ex vivo lung perfusion in clinical lung transplantation. $\mathrm{N}$ Engl J Med 2011;364:1431-40.

3. Steen S, Sjöberg T, Pierre L, et al. Transplantation of lungs from a non-heart-beating donor. Lancet 2001;357:825-9.

4. Roman MA, Nair S, Tsui S, et al. Ex vivo lung perfusion: a comprehensive review of the development and exploration of future trends. Transplantation 2013;96:509-18.

5. Oto T, Levvey BJ, Whitford H, et al. Feasibility and utility of a lung donor score: correlation with early posttransplant outcomes. Ann Thorac Surg 2007;83:257-63.

6. Loor G, Radosevich DM, Kelly RF, et al. The University of Minnesota Donor Lung Quality Index: A ConsensusBased Scoring Application Improves Donor Lung Use. Ann Thorac Surg 2016;102:1156-65.

7. Schiavon M, Marulli G, Rebusso A, et al. Normothermic Perfusion of Donor Marginal Lungs With the Organ Care System Lung: Clinical and Morphologic Evaluation. J Cardiothorac Vasc Anesth 2016;30:1032-7.

8. Schiavon M, Di Gregorio G, Marulli G, et al. Feasibility and Utility of Chest-X ray on Portable Normothermic Perfusion System. Transplantation 2016;100:e48-9.

9. Himmat S, Alzamil A, Aboelnazar N, et al. A Decrease in Hypoxic Pulmonary Vasoconstriction Correlates With Increased Inflammation During Extended Normothermic Ex Vivo Lung Perfusion. Artif Organs 2018;42:271-9.

10. Warnecke G, Moradiellos J, Tudorache I, et al. Normothermic perfusion of donor lungs for preservation and assessment with the Organ Care System Lung before bilateral transplantation: a pilot study of 12 patients. Lancet 2012;380:1851-8.

11. Warnecke G, Van Raemdonck D, Smith M, et al. The Organ Care System (OCS ${ }^{\mathrm{TM}}$ ) Lung INSPIRE International Trial Results. J Heart Lung Transplant 2015;34:S96.

12. Loor G, Warnecke G, Smith M, Kukreja J, et al. The OCS Lung EXPAND International Trial Interim Results. J Heart Lung Transplant 2016;35:S68-9.

13. Ceulemans LJ, Monbaliu D, Verslype C, et al. Combined liver and lung transplantation with extended normothermic lung preservation in a patient with end-stage emphysema complicated by drug-induced acute liver failure. Am J Transplant 2014;14:2412-6.

14. Cypel M, Yeung JC, Hirayama S, et al. Technique for 
prolonged normothermic ex vivo lung perfusion. J Heart Lung Transplant 2008;27:1319-25.

15. Loor G, Howard BT, Spratt JR, et al. Prolonged EVLP Using OCS Lung: Cellular and Acellular Perfusates. Transplantation 2017;101:2303-11.

16. Sommer W, Salman J, Avsar M, et al. Prediction of transplant outcome after 24-hour ex vivo lung perfusion using the Organ Care System in a porcine lung transplantation model. Am J Transplant 2018. [Epub ahead of print].

17. Nguyen AP, Gabriel RA, Golts E, et al. Severity of Acute Kidney Injury in the Post-Lung Transplant Patient Is

Cite this article as: Schiavon M, Zampieri D, Marulli G, Verderi E, Rebusso A, Comacchio GM, Nicotra S, Loy M, Lunardi F, Feltracco P, Calabrese F, Cozzi E, Rea F. Pushing the limits of reconditioning: extended normothermic lung perfusion in an extended criteria donor. J Thorac Dis 2018;10(12):E796E801. doi: 10.21037/jtd.2018.10.125
Associated With Higher Healthcare Resources and Cost. J Cardiothorac Vasc Anesth 2017;31:1361-9.

18. Warnecke G, Van Raemdonck D, Smith MA, et al. Normothermic ex-vivo preservation with the portable Organ Care System Lung device for bilateral lung transplantation (INSPIRE): a randomised, open-label, non-inferiority, phase 3 study. Lancet Respir Med 2018;6:357-67.

19. den Hengst WA, Gielis JF, Lin JY, et al. Lung ischemiareperfusion injury: a molecular and clinical view on a complex pathophysiological process. Am J Physiol Heart Circ Physiol 2010;299:H1283-99. 